\title{
A comparison of bow shock models with Cluster observations during low Alfvén Mach number magnetic clouds
}

\author{
L. Turc ${ }^{1}$, D. Fontaine ${ }^{1}$, P. Savoini ${ }^{1}$, H. Hietala ${ }^{2, *}$, and E. K. J. Kilpua ${ }^{2}$ \\ ${ }^{1}$ LPP, Ecole Polytechnique-CNRS-UPMC, route de Saclay, 91128 Palaiseau, France \\ ${ }^{2}$ Department of Physics, University of Helsinki, P.O. Box 64, 00014 Helsinki, Finland \\ *now at: Blackett Laboratory, Imperial College London, South Kensington, London SW7 2AZ, UK
}

Correspondence to: L. Turc (lucile.turc@lpp.polytechnique.fr)

Received: 18 October 2012 - Revised: 14 March 2013 - Accepted: 9 May 2013 - Published: 11 June 2013

\begin{abstract}
Magnetic clouds (MCs) are very geoeffective solar wind structures. Their properties in the interplanetary medium have been extensively studied, yet little is known about their characteristics in the Earth's magnetosheath. The Cluster spacecraft offer the opportunity to observe MCs in the magnetosheath, but before MCs reach the magnetosphere, their structure is altered when they interact with the terrestrial bow shock (BS). The physics taking place at the BS strongly depends on $\Theta_{\mathrm{Bn}}$, the angle between the shock normal and the interplanetary magnetic field. However, in situ observations of the BS during an MC's crossing are seldom available. In order to relate magnetosheath observations to solar wind conditions, we need to rely on a model to determine the shock's position and normal direction. Yet during MCs, the models tend to be less accurate, because the Alfvén Mach number $\left(M_{\mathrm{A}}\right)$ is often significantly lower than in regular solar wind. On the contrary, the models are generally optimised for high $M_{\mathrm{A}}$ conditions. In this study, we compare the predictions of four widely used models available in the literature (Wu et al., 2000; Chapman and Cairns, 2003; Jeřáb et al., 2005; Měrka et al., 2005b) to Cluster's dayside BS crossings observed during five MC events. Our analysis shows that the $\Theta_{\mathrm{Bn}}$ angle is well predicted by all four models. On the other hand, the Jeřáb et al. (2005) model yields the best estimates of the BS position during low $M_{\mathrm{A}}$ MCs. The other models locate the BS either too far from or too close to Earth. The results of this paper can be directly used to estimate the BS parameters in all studies of MC interaction with Earth's magnetosphere.
\end{abstract}

Keywords. Interplanetary physics (planetary bow shocks) magnetospheric physics (solar wind-magnetosphere interactions)

\section{Introduction}

Magnetic clouds (MCs) are a subset of coronal mass ejections characterised in the interplanetary medium by an increase of the interplanetary magnetic field (IMF) strength and a smooth rotation of its direction (Burlaga et al., 1981). They generally also present a lower proton density and temperature than the ambient solar wind. Because of the low density and the high IMF magnitude, the Alfvén Mach number $\left(M_{\mathrm{A}}\right)$ is low during MCs, falling below 5 and even approaching 1 in some extreme events (see, for example, Ridley, 2007).

Understanding how MCs interact with Earth's environment is of major interest for space weather, as they are known to trigger intense geomagnetic storms (Yermolaev et al., 2012; Huttunen et al., 2005, and references therein). One step in the complicated chain of events leading to such a storm is their encounter with the bow shock (BS), which alters the cloud structure. The modified MCs then propagate into the magnetosheath and interact with the magnetosphere. Their structure in the magnetosheath can be observed, for example, by Cluster (Escoubet et al., 1997), the Geomagnetic Tail Lab (GEOTAIL) (Nishida, 1994) or the Time History of Events and Macroscale Interactions during Substorms (THEMIS) (Angelopoulos, 2008) spacecraft, which occasionally cross this region, while Wind (Acuña et al., 1995) and the Advanced Composition Explorer (ACE) (Stone et al., 1998) spacecraft provide a continuous monitoring of the solar wind in Lagrangian point L1. However, we seldom have observations of the MCs when they cross the BS.

In order to relate downstream observations with solar wind conditions upstream of the BS, we need to know the BS position and shape. We can then calculate the obliquity angle, 
which is crucial for the shock's physics. It is defined as the angle between the normal to the BS surface at a given point and the IMF direction, and generally noted as $\Theta_{\mathrm{Bn}}$. When a spacecraft crosses the BS, the shock's position and obliquity can be determined locally. But if a large coverage of the BS surface is required, or if there is no observation in the vicinity of the BS, we have to rely on a model to estimate the shock parameters. However, although MCs are one of the most important storm drivers, most existing BS models are optimised for high $M_{\mathrm{A}}$ conditions.

In this study, we will focus on a few events where Cluster encounters the BS multiple times during an MC's crossing. The spacecraft provides us with quasi-simultaneous observations upstream and downstream of the shock, and information about the shock's position. We will use these events to compare the predictions of different BS models available in the literature with in situ observations, in order to test their reliability during $\mathrm{MC}$ events. The aim of this work is to select an existing tool on which we could rely to estimate the shock parameters in MC conditions.

\section{Models, data sets and methods}

\subsection{Bow shock models}

Since the prediction and the discovery of Earth's BS, many models have been developed in an attempt to reproduce its stand-off distance, shape and response to changes in solar wind parameters (e.g. see Měrka et al., 2003, for review). A large part of them is based on fits of observed BS crossings combined with gasdynamic or magnetohydrodynamic (MHD) considerations (e.g. Němeček and Šafránková, 1991; Peredo et al., 1995), whereas some others rely on MHD simulation results (e.g. Cairns and Lyon, 1995).

A comparative study of BS models has already been led by Měrka et al. (2003, 2005a), who compared the predictions of six different models to 5870 BS crossings observed by the IMP 8 spacecraft. As their data set covered a large range of solar wind parameters, they were able to test them under both normal and extreme solar wind conditions. We focus here on the BS position in the range of parameters relevant to MCs, i.e. at low Mach number. The study of Merka et al. (2005a) showed that in such conditions the Němeček and Šafránková (1991) model yields more consistent results than other models that have been specifically designed to account for Mach number dependence, such as the Farris and Russell (1994) or the Cairns and Lyon (1995) models.

On the basis of the conclusions of Merka et al. (2005a) and taking into account the new versions of the models that have been developed since, we will compare the predictions of the following models to BS crossings observed during MCs:

- The Jeřáb et al. (2005) model (hereafter referred to as J05) is an improvement of the Němeček and Šafránková (1991) model built upon a larger data set (5270 cross- ings instead of 21). Indeed, it has been stressed that the flaws of the Němeček and Šafránková (1991) model under normal solar wind conditions were due to the lack of data points (Měrka et al., 2005a). As the Němeček and Šafránková (1991) model, the J05 model explicitly depends on solar wind dynamic pressure and IMF strength, whereas the magnetosonic Mach number $\left(M_{\mathrm{ms}}\right)$ has been replaced by $M_{\mathrm{A}}$ in the new version (see Peredo et al. (1995) for discussion about these Mach numbers);

- The Chapman and Cairns (2003) model (CC03), based on MHD simulations, adds a flaring parameter depending on $M_{\mathrm{A}}$ to Cairns and Lyon (1995) BS stand-off distance estimate. The flaring parameter depended previously only on solar wind dynamic pressure, but the model predictions under low $M_{\mathrm{A}}$ conditions have been improved by adding a $M_{\mathrm{A}}$ dependence, as suggested by Cairns et al. (1995). There are in fact two versions of this model, corresponding to different values of the angle between the solar wind speed and the IMF, $\theta_{\mathrm{Bv}}: 90^{\circ}$ and $45^{\circ}$. The latter introduces a north-south asymmetry in the BS shape. In our study we will use one version per MC, according to the average value of $\theta_{\mathrm{Bv}}$ during this event (see the last column of Table 1);

- The Wu et al. (2000) model (Wu00) combines Farris and Russell (1994) BS stand-off distance estimate with Shue et al. (1997) magnetopause shape. It uses the same parameters as the magnetopause of Shue et al. (1997), and thus explicitly depends on the dynamic pressure and the $B_{\mathrm{Z}}$ component of the IMF. It also takes into account the upstream $M_{\mathrm{ms}}$, as does Farris and Russell (1994) BS stand-off distance. In their work, Wu et al. (2000) tested this model in the case of a single MC and it yielded good results for this specific case. As this event took place in 1995, before Cluster was launched, it was not included in our study which focuses on Cluster observations;

- The Měrka et al. (2005b) model (M05) is a correction to the Peredo et al. (1995) model. It is based on the same data set (550 crossings) and the same analysis method, and thus only depends on $M_{\mathrm{A}}$ and the dynamic pressure. Because of the few data points in the low $M_{\mathrm{A}}$ range, the uncertainty on the BS position is very large when $M_{\mathrm{A}}<5$, and Merrka et al. (2005b) recommended not to use their model for $M_{\mathrm{A}}<3$. However, the specific feature of this model is that the empirical $M_{\mathrm{A}}$ dependence is included in the coefficients of the equation describing the BS surface. It causes the BS shape to be much more variable than in other models. Moreover, this model does not rely on the formula of Spreiter et al. (1966) or its version modified by Farris and Russell (1994) to take into account the vanishing of the BS when the upstream Mach number reaches 1 . These formulas lead to the BS stand-off distance being proportional respectively to 
Table 1. Start and end time of the five low $M_{\mathrm{A}}$ events, observed BS crossings and solar wind parameters. The numbers between parentheses in the third column correspond to the number of BS crossings taking place when $M_{\mathrm{A}}<5$. The solar wind density, speed, IMF amplitude and $B_{\mathrm{Z}}$ component given in columns 5 to 8 are averaged over each event. The value of $\theta_{\mathrm{Bv}}$ given in column 9 refers to the version of the Chapman and Cairns (2003) model used in each event.

\begin{tabular}{|c|c|c|c|c|c|c|c|c|}
\hline Start time & End time & $\begin{array}{c}\text { BS } \\
\text { crossings }\end{array}$ & $M_{\mathrm{A}}$ & $\begin{array}{c}n \\
\left(\mathrm{~cm}^{-3}\right)\end{array}$ & $\begin{array}{c}V \\
\left(\mathrm{kms}^{-1}\right)\end{array}$ & $\begin{array}{r}B \\
\text { (nT) }\end{array}$ & $\begin{array}{r}B_{\mathrm{Z}} \\
(\mathrm{nT})\end{array}$ & $\theta_{\mathrm{Bv}}$ \\
\hline 19 Mar 2001 - 17:00 UT & 20 Mar 2001 - 01:00 UT & $11(8)$ & $3.3-9.7$ & 13 & 460 & 14.4 & -8.9 & $90^{\circ}$ \\
\hline 31 Mar 2001 - 17:00 UT & 31 Mar $2001-23: 00$ UT & $11(11)$ & $2.0-4.2$ & 13 & 610 & 27.1 & -13.6 & $90^{\circ}$ \\
\hline 22 Apr 2001 - 06:00 UT & 22 Apr 2001 - 13:00 UT & $7(7)$ & $3.6-3.9$ & 9.9 & 370 & 14 & -11.2 & $90^{\circ}$ \\
\hline 27 Jan $2003-00: 00$ UT & 27 Jan $2003-15: 00 \mathrm{UT}$ & $15(12)$ & $2.6-7.0$ & 3.1 & 530 & 10.5 & 6.7 & $45^{\circ}$ \\
\hline 14 Apr 2006 - 00:30 UT & 14 Apr 2006 - 09:30 UT & $9(9)$ & $2.3-3.3$ & 6.1 & 510 & 17.5 & -11 & $90^{\circ}$ \\
\hline
\end{tabular}

$1 / M_{\mathrm{A}}^{2}(\mathrm{CC} 03$ model $)$ and $1 /\left(M_{\mathrm{A}}^{2}-1\right)(\mathrm{Wu} 00$ and J05 models). The M05 model will be used as a means of comparison to illustrate the different $M_{\mathrm{A}}$ dependences.

Among the models presented above, only the M05 model gives explicit calculations of the variance in the predicted shock position. Thus, in the other considered models, the uncertainty of predictions is unknown. Although uncertainty estimations increase greatly a model's usability, we will apply the same analysis method to all four models.

As an example, the corresponding modelled BS shapes in quiet solar wind conditions ( $V=400 \mathrm{~km} \mathrm{~s}^{-1}, B=5 \mathrm{nT}, n=$ $6 \mathrm{~cm}^{-3}$ ) are displayed in Fig. 1 (solid lines - the data points will be discussed in the following part) in the $X-R$ plane, where $R=\sqrt{Y^{2}+Z^{2}}$ and $X, Y$ and $Z$ are given in the Geocentric Solar Ecliptic (GSE) reference frame. Wu00 and M05 (black and grey curves) BS nose positions are very similar, around 14 Earth radii $\left(R_{\mathrm{E}}\right)$. CC03 model (light blue) locates the subsolar point slightly farther, around $15 R_{\mathrm{E}}$, whereas $\mathrm{J} 05$ model (dark blue) locates it 2 to $3 R_{\mathrm{E}}$ closer to Earth than the other models.

In conclusion, the models take into account different solar wind parameters and have been built upon different data sets, which can be observations or simulation results. Even during normal solar wind conditions, they display evident differences, and too few models provide error bars.

\subsection{Used data}

\subsubsection{Cluster observations}

Cluster is a fleet of four spacecraft that orbit Earth and cross the different boundaries of its magnetic environment, with an orbital period of $57 \mathrm{~h}$. Their trajectory changes with time, in order to observe both dayside and nightside magnetosphere. They can regularly be found in the vicinity of the BS four to five months a year and provide us with direct observations of its position and normal direction.

When searching for events, our selection criteria were that Cluster encounters the shock multiple times during an MC while the $M_{\mathrm{A}}$ is below 5 . The selected events comprise long intervals of low $M_{\mathrm{A}}$ solar wind, at least a few hours, during which Cluster alternately moves from solar wind to the magnetosheath. The spacecraft observes roughly a dozen crossings per event. The crossings have to be clear enough to be well identified, especially when performing minimum variance analysis (MVA) to determine the BS normal direction.

During the active part of solar cycle 23 (2001-2006), in which events fulfilling our criteria were most likely to take place, we found five MCs during which Cluster crossed the BS several times while $M_{\mathrm{A}}$ was low $\left(M_{\mathrm{A}}<5\right)$. These events and their main characteristics are summarised in Table 1. Column 3 shows that most of the observed BS crossings take place under low $M_{\mathrm{A}}$. As it can be seen in columns 5 to 8 from the mean solar wind parameters, quite diverse upstream conditions were encountered. The IMF values remained always particularly high, as expected for MC events. It should be noted that these values averaged over each event are only given as general information about our data set. The solar wind parameters which will be used as inputs for the BS models are averaged over five minutes.

We did find a sixth event which also met our criteria: the 15 May $2005 \mathrm{MC}$, one of the most extreme solar wind events of solar cycle 23. The IMF strength reached values as high as $60 \mathrm{nT}$, while the density fell to $1 \mathrm{~cm}^{-3}$, causing $M_{\mathrm{A}}$ to drop around 1. During this event, none of the models studied here yields good estimates of the BS geocentric distance. Cluster encountered the shock between 15 and $20 R_{\mathrm{E}}$, whereas the models predict it to remain much farther sunward. For $13 \mathrm{~h}$, the BS-estimated position displays large fluctuations, sometimes moving outward beyond $50 R_{\mathrm{E}}$. The average distance between the BS position observed at Cluster's crossings and its estimated position ranges between $7 R_{\mathrm{E}}$ (M05 model) and $26 R_{\mathrm{E}}$ (J05 model). On the contrary, during the cases in Table 1 , its position is correctly estimated by at least some of the models. As a means of comparison, the average distance between observed and predicted $\mathrm{BS}$ ranges between $1 R_{\mathrm{E}}$ (Wu00 model) and $3 R_{\mathrm{E}}$ (M05) during the 27 January 2003 $\mathrm{MC}$, which will be discussed in Sect. 3. In the following, we will limit our study to the five MCs listed in Table 1.

Since we cannot describe all the events here in detail, we will use in following sections the 27 January $2003 \mathrm{MC}$ as an 


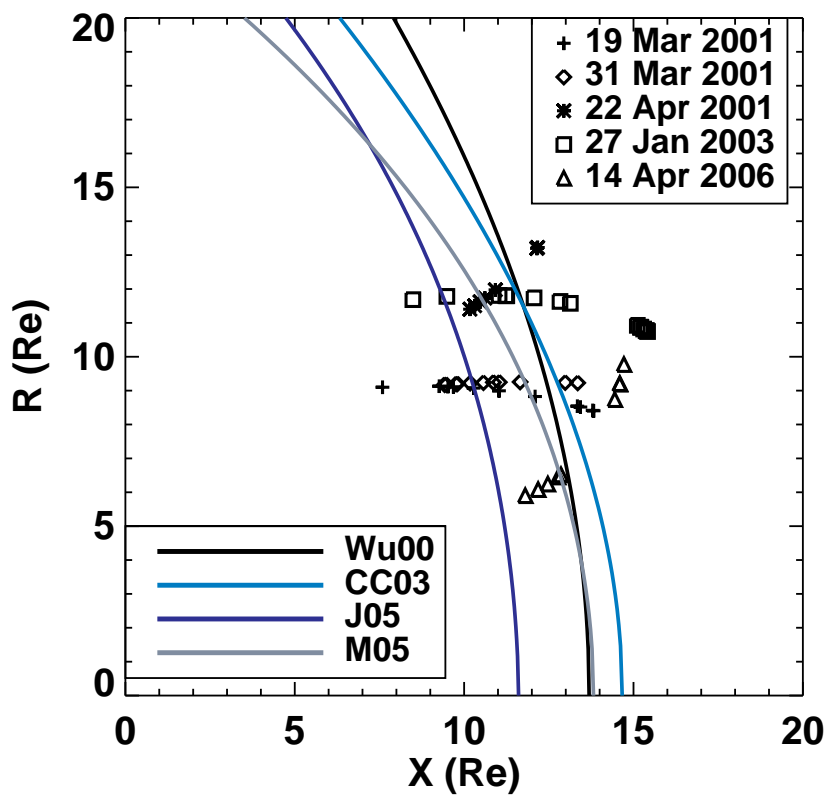

Fig. 1. Bow shock crossings data set and bow shock modelled shape in the $X-R$ plane - symbols: Cluster's crossings during the studied events - solid lines: modelled bow shock shape under quiet solar wind conditions $\left(V=400 \mathrm{~km} \mathrm{~s}^{-1}, B=5 \mathrm{nT}, n=6 \mathrm{~cm}^{-3}\right)$.

example, as it presents a wide variety of BS crossings. $M_{\mathrm{A}}$ ranges from 2 to 11 and remains below 5 during most of the MC passage.

Although Cluster provides us with four simultaneous data sets of solar wind parameters, we will only use measurements from a single spacecraft, since adding the other ones does not bring further information suitable for this study.

Cluster $1 \mathrm{BS}$ crossings in the $X-R$ plane are shown in Fig. 1; each type of symbols corresponds to a different event. The crossings have been identified using magnetic field and electron data, respectively from the FluxGate Magnetometer (FGM) and the Plasma Electron And Current Experiment (PEACE) (Balogh et al., 1997; Johnstone et al., 1997). These crossings are located in the dayside region, from 7 to $16 R_{\mathrm{E}}$ in the x-direction and from 5 to $14 R_{\mathrm{E}}$ along $R$. Cluster's orbit varies with time, and its apogee is around $19 R_{\mathrm{E}}$, so the studied events cover the largest range of shock crossings that can be reached by this spacecraft. When comparing these data points to the average BS shapes given by the models, we notice that the BS has been observed far from its usual position.

\subsubsection{Solar wind inputs}

The unperturbed solar wind upstream of Earth is observed by several spacecraft whose measurements can be used as inputs for the BS models. Since the Wind spacecraft is located far from the Sun-Earth line during the first three events listed in Table 1, the ACE spacecraft is preferred as a solar wind proxy because it provides us with consistent data sets throughout the five studied MCs. Though Cluster regularly observes the solar wind close to the BS, its measurements cannot be used as model inputs in our study because our analysis requires continuous solar wind measurements, as will be detailed in Sect. 2.3.

The OMNI data set, available at NASA's Coordinated Data Analysis Web (CDAWeb), is not used for the same reason as Wind: it is built upon different spacecraft measurements for the time intervals listed in Table 1. During the first four MCs, it is based on ACE observations, but during the last event, 14 April 2006, data are taken from Wind measurements. However, when Wind or OMNI data are also available, they may be used as a means of comparison.

An example of observations from the four data sets mentioned above, ACE, Wind and Cluster 1 observations and the 1 min-resolution OMNI data which correspond here to ACE data propagated to the BS nose, is shown in Fig. 2. It displays magnetic field measurements during the $15 \mathrm{~h}$ of the 27 January $2003 \mathrm{MC}$ when the $M_{\mathrm{A}}$ was low. ACE and Wind measurements have been shifted in time by respectively 45 and $35 \mathrm{~min}$, to fit Cluster 1 time. The IMF direction is given in the GSE frame to which we associated a spherical coordinate system. $\theta$ is here the colatitude, ranging between 0 and $180^{\circ}$ and $\varphi$ the longitude, ranging between -180 and $180^{\circ}$.

The three solar wind data sets (i.e. ACE, Wind and OMNI) display very similar features. As for Cluster 1, it encounters the shock 15 times, and alternately moves from solar wind to the magnetosheath. The shock crossings take place at each sharp variation of the IMF amplitude shown in the first panel of Fig. 2. When Cluster 1 observes an IMF strength several times higher than the other spacecraft, it is located in the magnetosheath. However, whenever Cluster 1 is in the solar wind, it appears clearly that the IMF amplitude and direction are similar on the four data sets.

In the following, we use data from the ACE Magnetic Field Experiment (MAG) and Solar Wind Electron Proton Alpha Monitor (SWEPAM) (Smith et al., 1998; McComas et al., 1998) as inputs for the BS models. These data are shifted in time from L1 to the BS, assuming that the solar wind propagates along the Sun-Earth line at a constant speed and that the structures do not change during their propagation. For each event, we determine the time shift that will be applied to the entire data set using the measured solar wind speed averaged over the whole event. On the basis of the maximum and minimum speed observed during the interval, we estimated that using a constant time shift throughout an entire event leads to errors of $\pm 5 \mathrm{~min}$ on the propagation time. As our data are averaged over $5 \mathrm{~min}$, a constant time shift is a reasonable approximation.

As mentioned in Sect. 2.1, the Wu00 model depends on the magnetosonic Mach number, whereas the other models take into account the Alfvén Mach number. The $M_{\mathrm{ms}}$ is calculated using ACE radial proton temperature measurements and assuming an isotropic temperature. Whenever Wind data were also available near L1, it has been checked that the results 


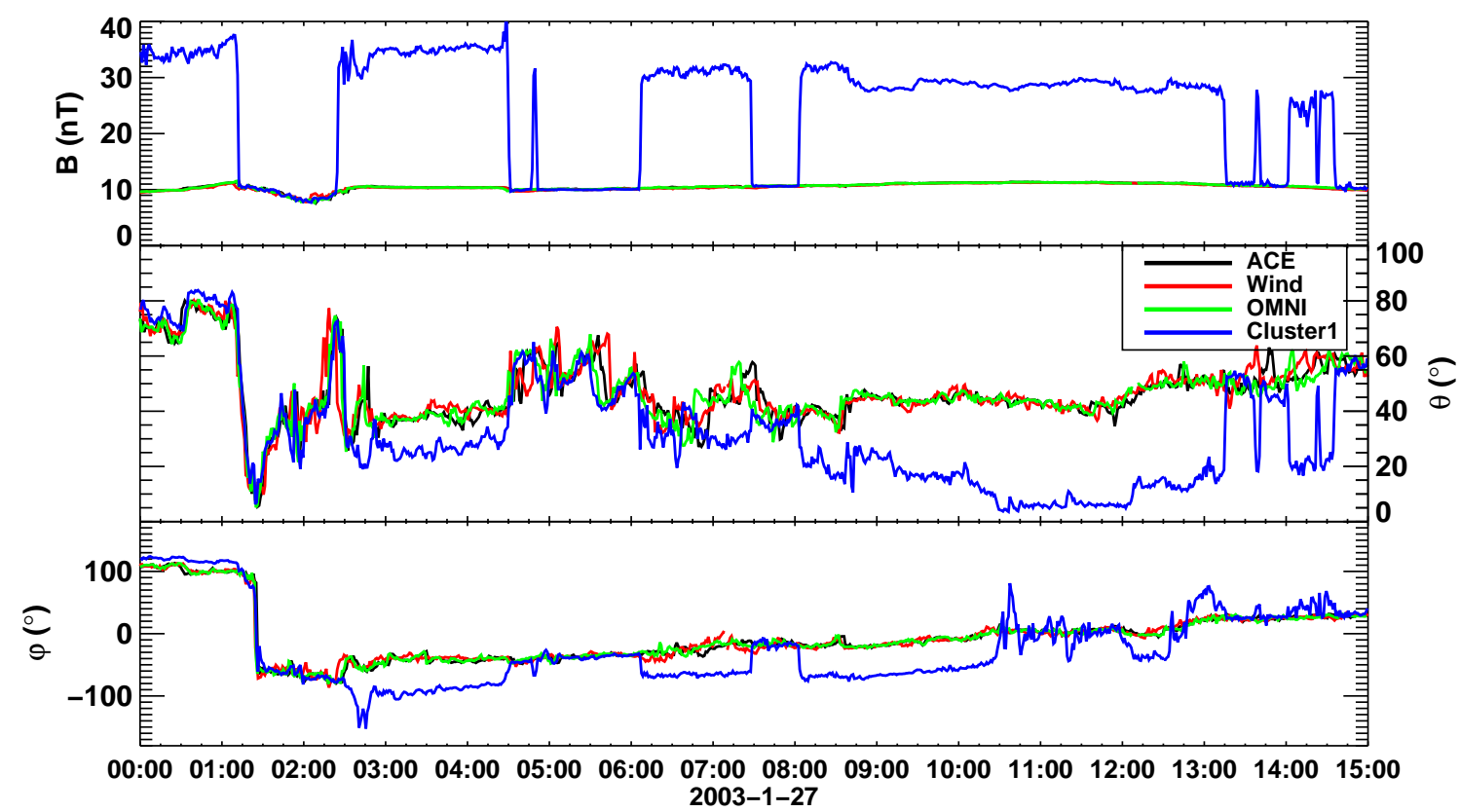

Fig. 2. Magnetic field amplitude and direction during the 27 January 2003 MC from ACE (black), Wind (red), OMNI (green) and Cluster 1 (blue) data sets - see text for definition of $\theta$ and $\varphi$.

are not sensitive to the electron temperature. Since during MCs the IMF strength is high and the temperature is low, the Alfvén and magnetosonic Mach numbers are roughly equal in such solar wind conditions. In the following sections, we will only display and discuss the $M_{\mathrm{A}}$ dependence.

\subsection{Evaluation methods}

In previous studies comparing the predictive capabilities of different BS models (e.g. Merka et al., 2005a), the authors tried to match each observed BS crossing with a predicted one. However, since we have no information about the uncertainty of the predictions, except for the M05 model, we cannot set a validity range for the different model estimates. We use here another method to compare their accuracy, based on the plasma region, solar wind or magnetosheath, in which the spacecraft travels at a given time. Observations clearly show in which region Cluster 1 is located, because the plasma and magnetic field properties are distinctly different in the solar wind and in the magnetosheath (see the first panel of Fig. 2).

Even though we do not know the accurate BS position, we have information about its location relative to the spacecraft. We will then compare the estimated shock position with Cluster 1 location throughout the event and check if their predicted relative locations are consistent with the observations. We need to know the estimated position of the BS during the whole studied interval, hence our choice for ACE continuous solar wind measurements.

In order to compare quantitatively the models, we determine for each data point in which region each model predicts the spacecraft to be. If the predicted region corresponds to the region where the satellite is located, then we consider that the model is correct for this data point. If not, then the model is incorrect. This allows us to calculate rates of success and failure in both regions, solar wind and magnetosheath, for each model. The results of this analysis are presented in Sect. 3.1.

A key parameter to study the BS, besides its position, is $\Theta_{\mathrm{Bn}}$, defined as the angle between the IMF and the normal to the shock surface at a given point. The IMF direction is given by the solar wind monitor (e.g. ACE in our case). To determine the BS normal, we calculate the normal direction predicted by each model at the point where the Earth-Cluster line intersects the BS surface. It corresponds to the BS closest point to the spacecraft since Cluster is not located far on the flanks during these events (see Fig. 1). The shock obliquity is then determined using the ACE magnetic field propagated to Earth.

To compare these modelled shock obliquity to observations, we perform minimum variance analysis (MVA) on Cluster 1 magnetic field data. The minimum variance axis corresponds to the BS normal direction, as the normal component of the IMF is conserved when crossing the BS, according to Rankine-Hugoniot relations. We assume that MVA results are reliable when the eigenvalues of the diagonalised matrix are well distinct and when the normal direction does not depend on the time interval on which MVA is performed. To calculate the obliquity angle, we use upstream IMF values measured by Cluster 1 in the vicinity of the BS. The comparison between observed and estimated $\Theta_{\mathrm{Bn}}$ is presented in Sect. 3.2. 


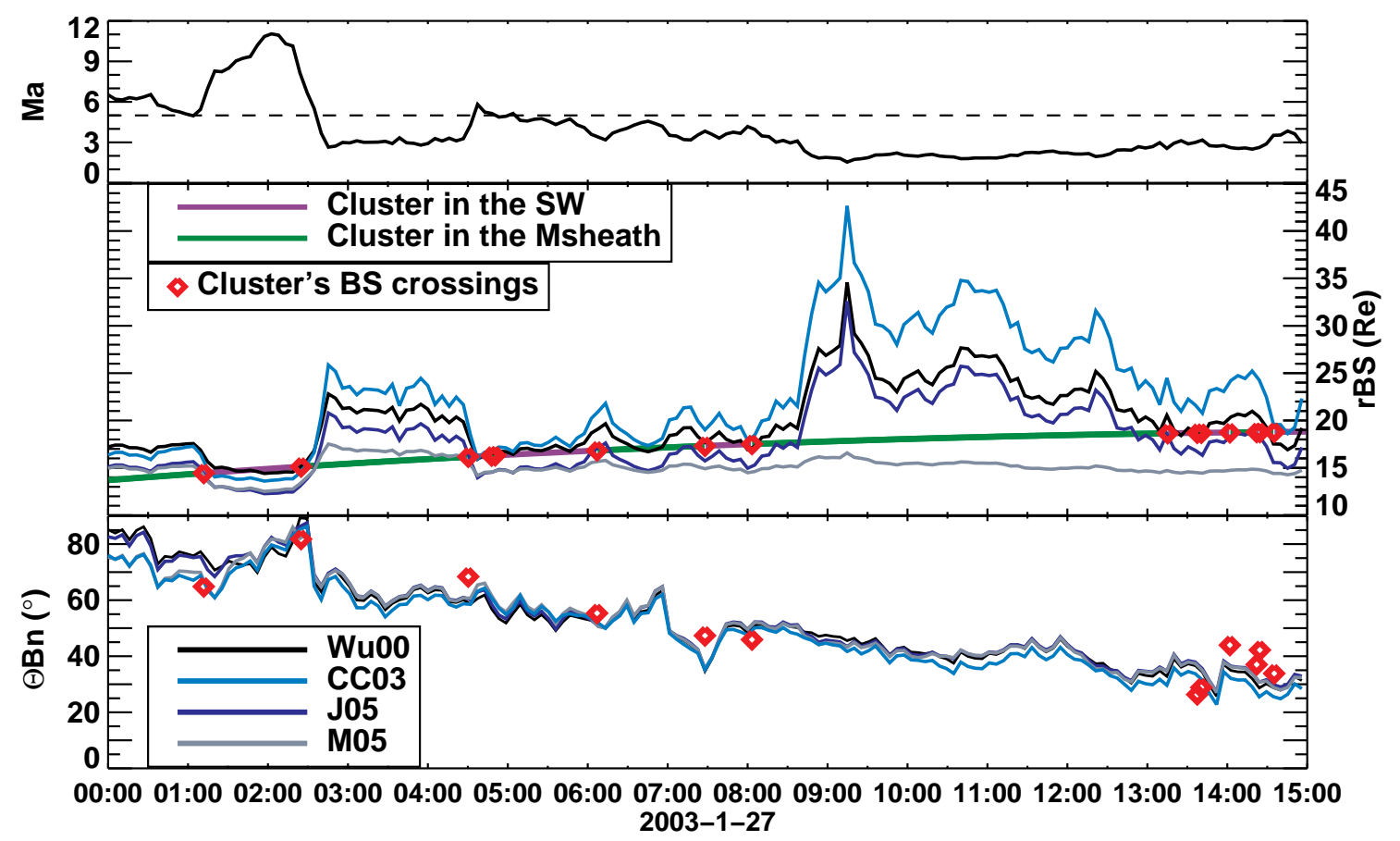

Fig. 3. Bow shock position and obliquity during the 27 January 2003 MC: models and observations - first panel: upstream Alfvén Mach number calculated from ACE data (time shift: $45 \mathrm{~min}$ ) - second panel: predicted time variation of the bow shock geocentric distance in Cluster's direction and Cluster's trajectory - third panel: predicted time variation of $\Theta_{\mathrm{Bn}}$ at Cluster's location - red diamonds: Cluster's bow shock crossings.

\section{Results}

\subsection{Bow shock position}

The variation of the BS geocentric distance, along the EarthCluster line, during the 27 January 2003 MC is shown in Fig. 3 (second panel) for the four models. From 00:00 UT to 09:00 UT, the four models display the same features, which are anti-correlated to the changes in the upstream $M_{\mathrm{A}}$ (Fig. 3, first panel) and dynamic pressure (not shown but similar to $\left.M_{\mathrm{A}}\right)$. Even if the Wu00 model does not depend on $M_{\mathrm{A}}$ but on $M_{\mathrm{ms}}$, its variations are similar to the other models, as expected from $\mathrm{MC}$ conditions.

In $\mathrm{Wu} 00, \mathrm{CC} 03$ and J05 models, we also notice that the lower the Mach number is, the larger the fluctuations of the BS position are. This is in agreement with the $\mathrm{Wu}$ et al. (2000) observation that the BS position depends more sensitively on small changes in $M_{\mathrm{A}}$ when its values are very low because of the $1 / M_{\mathrm{A}}^{2}$ or $1 /\left(M_{\mathrm{A}}^{2}-1\right)$ dependence of the BS stand-off distance.

The fast BS dynamics seem to be mostly driven by the upstream $M_{\mathrm{A}}$, since we observe that the four models inversely follow its variations (see the first and second panels of Fig. 3; the behaviour of the M05 model after 09:00 UT will be discussed below). However, the amplitude of the response to the $M_{\mathrm{A}}$ fluctuations differs from one model to another. Indeed, the equations that govern the BS shape and position do not depend on $M_{\mathrm{A}}$ in exactly the same way, though often quite similarly as mentioned earlier. They also take into account different fitting parameters, specific to each model. Moreover, the J05 and Wu00 models explicitly depend on $B$ and $B_{\mathrm{Z}}$ respectively. The specific features of each model lead to different amplitudes of the response of the BS position to changes in the upstream solar wind parameters.

Besides the amplitude of the fluctuations in the BS position, there is another major difference between the models: their relative position. $\mathrm{Wu} 00$ and $\mathrm{CC} 03$ are found farther from Earth than M05 and J05. This trend remains until the end of this event, and is also observed during the other studied MCs for the CC03, Wu00 and M05 models. On the contrary, J05 position with respect to the other models varies during some of the events, though it is not observed in the case shown here.

Starting shortly before 09:00 UT and until the end of this event, the M05 model estimates, which were previously as accurate as the others, display completely different features, showing only a smooth decrease while the others display large fluctuations. These discrepancies are due to the fact that the second part of this event falls beyond the M05 model validity range, as $M_{\mathrm{A}}$ remains below 3 . However, it is interesting to notice that the M05 model presents less variability than the others in the low $M_{\mathrm{A}}$ range because the BS stand-off distance is not directly proportional to $1 /\left(M_{\mathrm{A}}^{2}-1\right)$ or $1 / M_{\mathrm{A}}^{2}$, unlike the other models. The Mach number dependence is 
included in the shock's shape parameters and causes a large flaring of the flanks, but the BS nose position is less sensitive to small $M_{\mathrm{A}}$ fluctuations. In contrast, the $\mathrm{CC} 03$ model displays the largest variations, retreating more than $40 R_{\mathrm{E}}$ sunward (see Fig. 3 around 09:15 UT).

The green/purple line in the second panel of Fig. 3 corresponds to Cluster 1 trajectory during the 27 January 2003 event. On the basis of the spacecraft plasma and magnetic field observations, its orbit is drawn in green when Cluster 1 is in the magnetosheath, and in purple in the solar wind. The shock crossings are indicated as red diamonds. When the curve of the estimated BS distance from a given model is above Cluster 1 trajectory, the model predicts the spacecraft to be in the magnetosheath. Conversely, when the modelled BS distance is below the spacecraft orbit, the model predicts the spacecraft to be in the solar wind. We can then compare the model predictions with the actual location of the spacecraft relative to the $\mathrm{BS}$.

As detailed previously in Sect. 2.3, we calculate rates of success and failure in both regions, solar wind and magnetosheath, for each model and for each event. The results of this analysis over the five events are summarised in Table 2. The second and third columns are the success rates of the models in the solar wind and in the magnetosheath. The last column corresponds to the percentage of wrong predictions in both regions. As it is calculated over the entire data set, whereas the success rates take only into account either the solar wind or the magnetosheath part, their sum is not equal to $100 \%$.

The success rates show without ambiguity that three out of the four models locate the BS either too far (CC03 and $\mathrm{Wu} 00$ ) from or too close (M05) to Earth during these low $M_{\mathrm{A}}$ events. They have a high success rate in one of the regions but fail completely in the other one. The Wu00 model yields slightly better results, but still remains unreliable, since half of its predictions in the solar wind are incorrect. These three models also have a larger error rate than J05, which is another way to illustrate the fact that their predictions are less accurate. In the case of M05, this conclusion was expected because Měrka et al. (2005b) already pointed out that their model underestimates the BS distance in the subsolar region for $M_{\mathrm{A}}<5$.

On the other hand, the J05 model has high success rates both in the magnetosheath and in the solar wind, which means that its predictions agree on average quite well with Cluster 1 observations. We still have to keep in mind that it should be used carefully when it comes to really extreme events such as the 15 May 2005 MC, but during regular MCs this model predicts reasonably well the BS position.

\subsection{Bow shock normal and shock obliquity}

The variation of the $\Theta_{\mathrm{Bn}}$ estimated by the four models during the 27 January $2003 \mathrm{MC}$ is displayed in the lowest panel of Fig. 3. According to the models, the obliquity angle de-
Table 2. Success and error rates of the models' predictions during the five MC events.

\begin{tabular}{cccc}
\hline Model & $\begin{array}{c}\text { \% of success } \\
\text { (solar wind) }\end{array}$ & $\begin{array}{c}\text { \% of success } \\
\text { (magnetosheath) }\end{array}$ & \% of error \\
\hline Wu00 & 50 & 94 & 30 \\
CC03 & 36 & 98 & 36 \\
J05 & 90 & 73 & 18 \\
M05 & 99 & 23 & 36 \\
\hline
\end{tabular}

creases smoothly over the $15 \mathrm{~h}$ of data from 90 to $40^{\circ}$, slowly changing from a quasi-perpendicular shock to an oblique shock. It appears that the four models yield the same $\Theta_{\mathrm{Bn}}$ values and variation, the largest discrepancy being less than $10^{\circ}$ during the first hour.

The values of $\Theta_{\mathrm{Bn}}$ observed by Cluster 1 at its BS crossings are also indicated in the lowest panel of Fig. 3 as red diamonds. However, there are less observed BS crossings in the third panel than in the second panel of Fig. 3, 12 instead of 15 . Some crossings were discarded because MVA did not yield satisfactory results: the eigenvalues of the diagonalised matrix were not well distinct and/or the normal direction depended on the time interval on which MVA is performed. The observed $\Theta_{B n}$ values display a similar decrease from quasi-perpendicular to oblique shock, and their values are very close to the models' predictions. The discrepancies stay below $10^{\circ}$.

During the other MC events we studied, the predicted $\Theta_{\mathrm{Bn}}$ values are also in good agreement with MVA results, the discrepancies between the different models and between the models and the observations never exceeding $20^{\circ}$. The four models lead to similar results and predict very well the shock obliquity.

\section{Discussion and conclusions}

In this study we have compared the predictions of four different BS models to Cluster observations on the dayside during MCs, when the $M_{\mathrm{A}}$ was low. The variations of the modelled BS distances appear to be anti-correlated to changes in upstream $M_{\mathrm{A}}$ and dynamic pressure, which drive the global BS dynamics. However, the amplitude of these variations changes from one model to another because of the specific features inherent in each model. Another evident difference is their relative position, as some models always locate the shock closer to Earth than others. We then compared the BS distance estimates to Cluster observations and calculated how accurately the models predicted the spacecraft to be in the plasma region, solar wind or magnetosheath, where it actually was. This brought us to the conclusion that the Jeřáb et al. (2005) model yielded on average the best results.

It should be stressed that, unlike the other models studied here, the Jeřáb et al. (2005) model takes explicitly into 
account the IMF magnitude. The IMF strength might play an important part in determining the BS position when the $M_{\mathrm{A}}$ is low. This could be tested in future BS modelling, but it lies beyond the scope of this paper.

In the second part of our study, we focused on the BS normal direction and especially the obliquity angle $\Theta_{\mathrm{Bn}}$, and compared the model estimates with Cluster observations. It appeared that, unlike the BS distance, all models predict quite accurately the shock obliquity during the studied events.

In this work, although low $M_{\mathrm{A}}$ solar wind is not limited to MCs, we only considered MC events because of the geomagnetic activity they trigger. Outside MCs, a low $M_{\mathrm{A}}$ solar wind is generally due to low density and low speed, as it happened during the famous "day the solar wind almost disappeared", 11 May 1999, described, for example, by Farrugia et al. (2005). The $M_{\mathrm{A}}$ was low, but the IMF strength remained close to its usual value, and the dynamic pressure was very small because of the tenuous and slow solar wind. These conditions are quite different from MCs, where the low $M_{\mathrm{A}}$ is caused by a low density and high magnetic field amplitude, but the speed is generally high, and consequently the dynamic pressure is not so low. Moreover, the densities observed during MCs are not dramatically low, as it can be seen in the fifth column of Table 1.

The models not only depend on the $M_{\mathrm{A}}$, but also on the dynamic pressure, and in the case of the Wu et al. (2000) and the Jeřáb et al. (2005) models on the IMF $B_{\mathrm{Z}}$ component and strength, respectively. Low $M_{\mathrm{A}}$ solar wind outside MCs corresponds to different solar wind conditions, which may lead to different conclusions on the models' predictive capabilities. This could be the topic of a future analysis. Here we have focused on MCs because of their importance in driving activity in Earth's magnetosphere.

In MC studies focusing on the cloud interaction and alteration through the BS, the events with direct observations of MCs crossing the BS are seldom available. Most of the time, events are identified downstream in the magnetosheath, while the solar wind conditions are continuously monitored upstream by spacecraft at $\mathrm{L} 1$ or elsewhere. A model is therefore needed to relate downstream observations with upstream solar wind parameters and especially to estimate the shock configuration through $\Theta_{\mathrm{Bn}}$. In such a case, we suggest that one may use any of the models studied here with reasonable confidence to estimate the obliquity angle, and thus the shock configuration (quasi-perpendicular, oblique or quasiparallel). However, regarding the results on the BS position, the Jeřáb et al. (2005) model seems to be the most reliable for low $M_{\mathrm{A}}$ events and thus should be preferred to other models in the specific case of MCs.

Acknowledgements. The authors thank J. Merka for fruitful discussions on bow shock models, P. Robert for providing the MVA software and K. Andreeova for her advice on normal determination.
We thank the ACE SWEPAM and MAG instrument teams and the CDAWeb for providing the ACE data. We also acknowledge Cluster FGM and PEACE teams and the Cluster Active Archive for providing Cluster data.

Topical Editor R. Nakamura thanks J. Merka and one anonymous referee for their help in evaluating this paper.

\section{References}

Acuña, M. H., Ogilvie, K. W., Baker, D. N., Curtis, S. A., Fairfield, D. H., and Mish, W. H.: The Global Geospace Science Program and Its Investigations, Space Sci. Rev., 71, 5-21, doi:10.1007/BF00751323, 1995.

Angelopoulos, V.: The THEMIS Mission, Space Sci. Rev., 141, 534, doi:10.1007/s11214-008-9336-1, 2008.

Balogh, A., Dunlop, M. W., Cowley, S. W. H., Southwood, D. J., Thomlinson, J. G., Glassmeier, K. H., Musmann, G., Luhr, H., Buchert, S., Acuna, M. H., Fairfield, D. H., Slavin, J. A., Riedler, W., Schwingenschuh, K., and Kivelson, M. G.: The Cluster Magnetic Field Investigation, Space Sci. Rev., 79, 6591, doi:10.1023/A:1004970907748, 1997.

Burlaga, L., Sittler, E., Mariani, F., and Schwenn, R.: Magnetic loop behind an interplanetary shock - Voyager, Helios, and IMP 8 observations, J. Geophys. Res., 86, 6673-6684, doi:10.1029/JA086iA08p06673, 1981.

Cairns, I. H., Fairfield, D. H., Anderson, R. R., Carlton, V. E. H., Paularena K. I., and Lazarus A. J.: Unusual locations of Earth's bow shock on September 24-25, 1987: Mach number effects, J. Geophys. Res., 100, 47-62, doi:10.1029/94JA01978, 1995.

Cairns, I. H. and Lyon, J. G.: MHD simulations of Earth's bow shock at low Mach numbers: Standoff distances, J. Geophys. Res., 100, 17173-17180, doi:10.1029/95JA00993, 1995.

Chapman, J. F. and Cairns, I. H.: Three-dimensional modeling of Earth's bow shock: Shock shape as a function of Alfvén Mach number, J. Geophys. Res., 108, 1174, doi:10.1029/2002JA009569, 2003.

Escoubet, C. P., Schmidt, R., and Goldstein, M. L.: Cluster Science and Mission Overview, Space Sci. Rev., 79, 11-32, doi:10.1023/A:1004923124586, 1997.

Farris, M. H. and Russell, C. T.: Determining the standoff distance of the bow shock: Mach number dependence and use of models, J. Geophys. Res., 99, 17681, doi:10.1029/94JA01020, 1994.

Farrugia, C. J., Gratton, F. T., Gnavi, G., Matsui, H., Torbert, R. B., Fairfield, D. H., Ogilvie, K. W., Lepping, R. P., Terasawa, T., Mukai, T., and Saito, Y.: Magnetosheath waves under very low solar wind dynamic pressure: Wind/Geotail observations, Ann. Geophys., 23, 1317-1333, doi:10.5194/angeo-231317-2005, 2005.

Huttunen, K. E. J., Schwenn, R., Bothmer, V., and Koskinen, H. E. J.: Properties and geoeffectiveness of magnetic clouds in the rising, maximum and early declining phases of solar cycle 23, Ann. Geophys., 23, 625-641, doi:10.5194/angeo-23-625-2005, 2005.

Jeřáb, M., Němeček, Z., Šafránková, J., Jelínek, K., and Měrka, J.: Improved bow shock model with dependence on the IMF strength, Planet. Space Sci., 53, 85-93, doi:10.1016/j.pss.2004.09.032, 2005.

Johnstone, A. D., Alsop, C., Burge, S., Carter, P. J., Coates, A. J., Coker, A. J., Fazakerley, A. N., Grande, M., Gowen, R. A., Gur- 
giolo, C., Hancock, B. K., Narheim, B., Preece, A., Sheather, P. H., Winningham, J. D., and Woodliffe, R. D.: Peace: a Plasma Electron and Current Experiment, Space Sci. Rev., 79, 351-398, doi:10.1023/A:1004938001388, 1997.

McComas, D. J., Bame, S. J., Barker, P., Feldman, W. C., Phillips, J. L., Riley, P., and Griffee, J. W.: Solar Wind Electron Proton Alpha Monitor (SWEPAM) for the Advanced Composition Explorer, Space Sci. Rev., 86, 563-612, doi:10.1023/A:1005040232597, 1998.

Měrka, J., Szabo, A., Narock, T. W., King, J. H., Paularena, K. I., and Richardson, J. D.: A comparison of IMP 8 observed bow shock positions with model predictions, J. Geophys. Res., 108, 1077, doi:10.1029/2002JA009384, 2003.

Měrka, J., Szabo, A., Narock, T. W., Richardson, J. D., and King, J. H.: Three decades of bow shock observations by IMP 8 and model predictions, Planet. Space Sci., 53, 79-84, doi:10.1016/j.pss.2004.09.031, 2005a.

Měrka, J., Szabo, A., Slavin, J. A., and Peredo, M.: Threedimensional position and shape of the bow shock and their variation with upstream Mach numbers and interplanetary magnetic field orientation, J. Geophys. Res., 110, 4202, doi:10.1029/2004JA010944, 2005b.

Němeček, Z. and Šafránková, J.: The earth's bow shock and magnetopause position as a result of the solar wind-magnetosphere interaction, J. Atmos. Terr. Phys., 53, 1049-1054, 1991.

Nishida, A.: The GEOTAIL mission, Geophys. Res. Lett., 21, 28712873, doi:10.1029/94GL01223, 1994.

Peredo, M., Slavin, J. A., Mazur, E., and Curtis, S. A.: Threedimensional position and shape of the bow shock and their variation with Alfvénic, sonic and magnetosonic Mach numbers and interplanetary magnetic field orientation, J. Geophys. Res., 100, 7907-7916, doi:10.1029/94JA02545, 1995.
Ridley, A. J.: Alfvén wings at Earth's magnetosphere under strong interplanetary magnetic fields, Ann. Geophys., 25, 533-542, doi:10.5194/angeo-25-533-2007, 2007.

Shue, J.-H., Chao, J. K., Fu, H. C., Russell, C. T., Song, P., Khurana, K. K., and Singer, H. J.: A new functional form to study the solar wind control of the magnetopause size and shape, J. Geophys. Res., 102, 9497-9512, doi:10.1029/97JA00196, 1997.

Smith, C. W., L'Heureux, J., Ness, N. F., Acuña, M. H., Burlaga, L. F., and Scheifele, J.: The ACE Magnetic Fields Experiment, Space Sci. Rev., 86, 613-632, doi:10.1023/A:1005092216668, 1998.

Spreiter, J. R., Summers, A. L., and Alksne, A. Y.: Hydromagnetic flow around the magnetosphere, Planet. Space Sci., 14, 223 , doi:10.1016/0032-0633(66)90124-3, 1966.

Stone, E. C., Frandsen, A. M., Mewaldt, R. A., Christian, E. R., Margolies, D., Ormes, J. F., and Snow, F.: The Advanced Composition Explorer, Space Sci. Rev., 86, 1-22, doi:10.1023/A:1005082526237, 1998.

Wu, D. J., Chao, J. K., and Lepping, R. P.: Interaction between an interplanetary magnetic cloud and the Earth's magnetosphere: Motions of the bow shock, J. Geophys. Res., 105, 12627-12638, doi:10.1029/1999JA000265, 2000.

Yermolaev, Y. I., Nikolaeva, N. S., Lodkina, I. G., and Yermolaev, M. Y.: Geoeffectiveness and efficiency of CIR, sheath, and ICME in generation of magnetic storms, J. Geophys. Res., 117, A00L07, doi:10.1029/2011JA017139, 2012. 